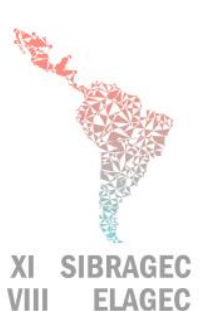

VIII ELAGEC

\section{SIMPÓSIO BRASILEIRO DE GESTÃO E ECONOMIA DA CONSTRUÇÃO}

VIII ENCUENTRO LATINOAMERICANO DE GESTIÓN Y ECONOMÍA DE LA CONSTRUCCIÓN

Do conhecimento à ação: práticas avançadas de gestão da produção Londrina, Paraná, Brasil. 23 a 25 de Outubro de 2019

\title{
ANÁLISE COMPARATIVA DO EMPREGO DO MÉTODO EVOLUTIVO PARA AVALIAÇÃO DE IMÓVEIS POR MEIO DE ESTIMATIVA DE CUSTOS E ORÇAMENTO ANALÍTICO
}

\author{
BINOTTO, Natália (1); BRESSIANI, Lucia (2); HIRANO, Eduardo Lovera (3); \\ SAVARIS, Gustavo (4)
}

(1) Universidade Tecnológica Federal do Paraná, (45)3039-6800, e-mail: nataliabinotto@ hotmail.com

(2) Universidade Tecnológica Federal do Paraná, e-mail: bressiani@utfpr.edu.br, (3) Universidade

Tecnológica Federal do Paraná, e-mail: ehirano@alunos.utfpr.edu.br, (4) Universidade Tecnológica Federal do Paraná, e-mail: gsavaris@utfpr.edu.br

\begin{abstract}
In the present research, the evolutionary method was applied to evaluate a residential building of four floors in the city of Toledo, Paraná. Thus, the purpose of the study was to verify and compare the difference in the final value of the property, through the use of different methodologies for budget realization. For this, three budget techniques were used: analytical budget, cost estimating using the $C U B$ category PP - 4, corrected by the addition of existing and not considered costs in the standard project and, finally, estimation by the use of the CUB classification $R 8-N$, corrected by a coefficient according to the constructive typology. Finally, it was possible to perform comparative analysis of the data, verifying the percentages and the best budgeting alternative for the building under study evaluation. As a result, the average values of the property were obtained for the analytical budgets, CUB PP -4 and $C U B R 8-N$, respectively: $R \$ 2.089 .512 .59, R \$ 2.214 .370 .01, R \$ 2.29 ., 789,62$. Therefore, it was found that in both cases, the CUB overestimates the construction value.
\end{abstract}

Keywords: Evolutionary method, Real estate evaluation, Analytical budget, CUB, Terrain evaluation.

\section{INTRODUÇÃ̃O}

Considerando que o imóvel é o bem de maior importância adquirido pelo homem, fica evidente a importância da precisão a ser obtida numa avaliação. E essa avaliação tem impacto para diversos setores como prefeituras, Receita Federal, agentes financiadores, dentre outros (FLORÊNCIO, 2011).

A NBR 14653-1 (ABNT, 2001) determina que sempre que possível a avaliação de um imóvel seja realizada pelo método comparativo direto de dados de mercado. Porém, muitas vezes as condições do mercado não permitem a utilização deste método, em função da inexistência de imóveis semelhantes a venda no mercado para comparação. Neste sentido, o método evolutivo se apresenta como uma opção para identificação do valor do imóvel.

O método evolutivo permite a obtenção do valor do imóvel pela soma das parcelas do valor do terreno e o custo da edificação. Porém, a forma como as avaliações estão sendo

BINOTTO, N.; BRESSIANI, L.; HIRANO, E. L.; SAVARIS, G. Análise comparativa do emprego do método evolutivo para avaliação de imóveis por meio de estimativa de custos e orçamento analítico. In: SIMPÓSIO BRASILEIRO DE GESTÃO E ECONOMIA DA CONSTRUÇÃO, 11., 2019, Londrina.

Anais [...]. Porto Alegre: ANTAC, 2019. Disponível em:

https://www.antaceventos.net.br/index.php/sibragec/sibragec2019/paper/view/471 
realizadas na atualidade com o uso deste método, pode resultar em valores que não representam a realidade do imóvel (COELHO, 2007).

Embora estimativas de custo por meio do uso do CUB (Custo Unitário Básico) devem ser utilizadas apenas na fase de viabilidade dos empreendimentos (MATTOS, 2006), profissionais da área de avaliações geralmente recorrem a este indicador para determinação do custo da edificação no método evolutivo.

Desta forma, o objetivo deste trabalho foi comparar a avaliação de um imóvel, por meio do método evolutivo, empregando duas técnicas para determinar o custo do mesmo, ou seja, com base no CUB e orçamento analítico. Para isso foi realizado um estudo de caso em um edifício residencial de quatro pavimentos na cidade de Toledo, Paraná, apresentando os valores obtidos na avaliação por meio dos dois métodos.

\section{REVISÃO DA LITERATURA}

O método evolutivo, segundo a NBR 14653-2 (ABNT, 2001), determina o valor do imóvel pelo somatório de suas partes (valor do terreno e custo da construção). É aplicado preferencialmente a bens urbanos que não possuem outros imóveis similares em número suficiente para que se possam utilizar outros métodos avaliativos.

A referida norma preconiza graus de fundamentação, que correspondem ao aprofundamento da pesquisa do profissional de avaliação. Esses graus são divididos em níveis I, II e III, sendo esse último o de maior aprofundamento. O Quadro 1 apresenta um dos itens levados em consideração na determinação do grau de fundamentação, que é a estimativa de custo, e como ela é enquadrada em função de cada grau.

\section{Quadro 1 - Grau de fundamentação para quantificação do custo}

\begin{tabular}{|c|c|c|c|}
\hline \multirow[t]{2}{*}{ Descrição } & \multicolumn{3}{|c|}{ Graus } \\
\hline & III & II & I \\
\hline $\begin{array}{l}\text { Estimativa } \\
\text { custo direto }\end{array}$ & $\begin{array}{ll}\text { Pela elaboração do } \\
\text { orçamento, } & \text { no } \\
\text { mínimo sintético } & \end{array}$ & \begin{tabular}{llr} 
& \multicolumn{2}{c}{ Pela utilização de } \\
custo unitário básico \\
para & projeto \\
semelhante ao projeto \\
padrão
\end{tabular} & $\begin{array}{l}\text { Pela utilização de } \\
\text { custo unitário básico } \\
\text { para projeto diferente } \\
\text { do projeto padrão, com } \\
\text { os devidos ajustes }\end{array}$ \\
\hline
\end{tabular}

Fonte: Adaptado de NBR 14653-2 (ABNT, 2001)

A estimativa realizada por uma das três formas apresentada no Quadro 1, é utilizada para determinar o custo da construção, que será somado ao valor do terreno para obter o valor do imóvel avaliado.

Embora a NBR 14653-2 (ABNT, 2001) especifique que a determinação do custo possa ser realizada por qualquer uma das três formas citadas, a maior pontuação é atribuída ao uso do orçamento no mínimo sintético (Grau III). Isso porque sua precisão é maior que as outras estimativas apresentadas no Quadro 1.

A literatura apresenta algumas pesquisas que compararam o orçamento detalhado a outros métodos de orçamentação. Neste contexto podem ser destacados os trabalhos de Catanhede e Schmitt (2003), Fernandes et al. (2017) e Knolseisen, Librelotto e Jungles (2001), que realizaram análises comparativas de estimativa de custo utilizando o CUB e orçamento analítico. Os resultados mostraram que os custos estimados com o CUB 
foram maiores, podendo ser até $25 \%$ superiores quando esse indicador é utilizado sem considerar os custos não contemplados pelo mesmo, como instalações especiais, ajardinamento, dentre outros.

\section{PROCEDIMENTOS METODOLÓGICOS}

Esta pesquisa apresenta um estudo de caso sobre a avaliação de um imóvel, por meio do método evolutivo, considerando duas formas de identificar o custo da edificação, ou seja, orçamento detalhado e estimativa por meio do CUB. A Figura 1 apresenta o fluxograma com as etapas da pesquisa.

\section{Figura 1 - Etapas da pesquisa}

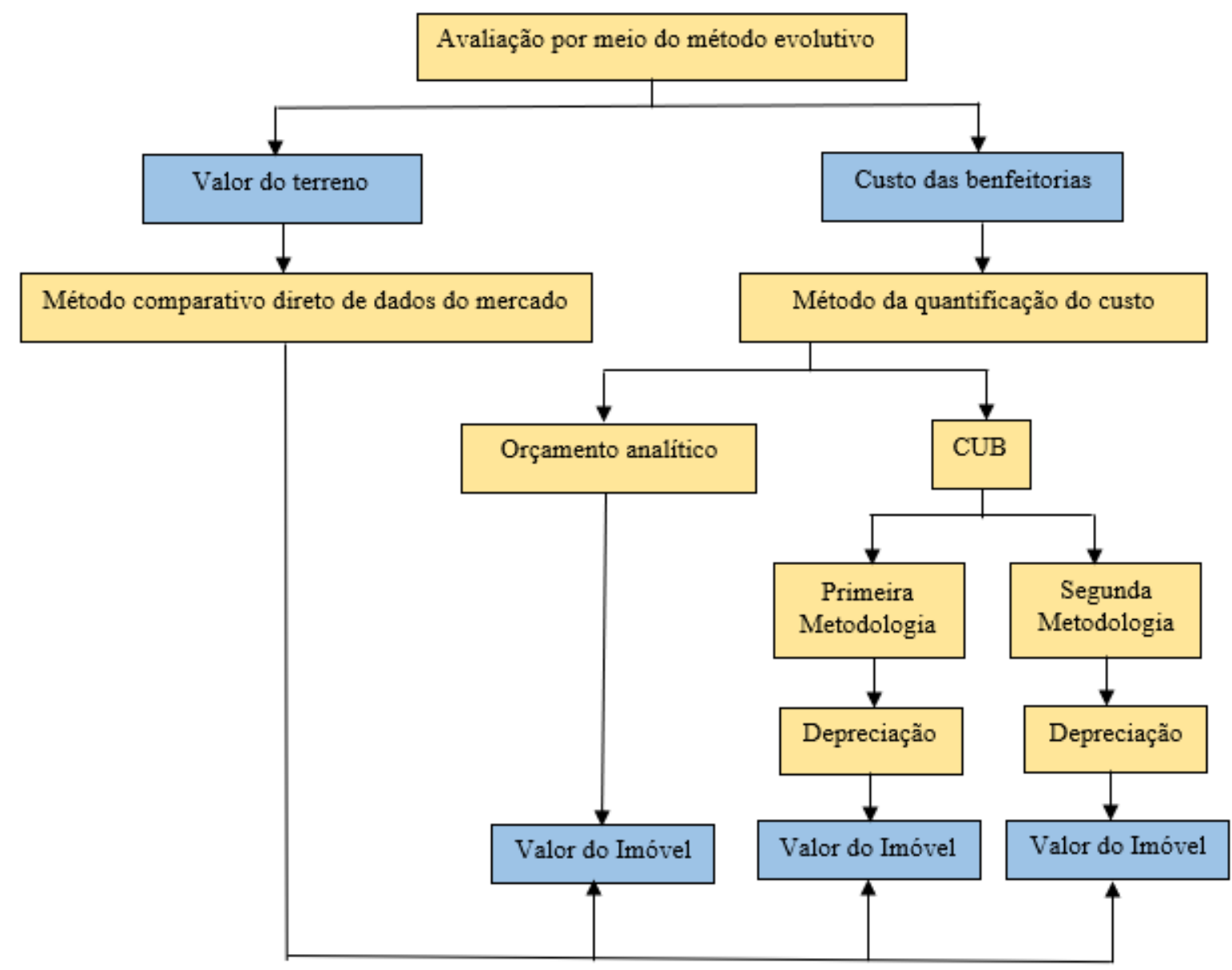

Fonte: AUTORES (2019)

O empreendimento do estudo de caso se trata de um edifício residencial, de quatro pavimentos de $1075 \mathrm{~m}^{2}$ em um terreno de $780 \mathrm{~m}^{2}$. Possui 16 apartamentos de $60 \mathrm{~m}^{2}$ cada um, com dois quartos, banheiro social, sala, cozinha, lavanderia, sacada e uma vaga de garagem.

Para avaliação do imóvel foi utilizado o método evolutivo, que considera o valor do terreno e o custo de reprodução das benfeitorias e aplicação de um fator de comercialização. Para a determinação do valor do terreno e o custo das benfeitorias foi preciso recorrer a outros métodos de avaliação, como descrito a seguir. 


\subsection{Valor do terreno}

Para avaliação do terreno foi utilizado o método comparativo direto de dados de mercado. Para isso, foram analisados 20 terrenos similares ao do estudo de caso, que estavam à venda ou tinham sido vendidos até seis meses antes da coleta de dados. Em seguida, os dados foram homogeneizados e saneados, por meio do tratamento por fatores. Os fatores considerados foram: oferta, frente, área, localização, topografia e melhoramentos públicos (existência de calçada, meio fio, asfalto na rua, dentre outros).

\subsection{Custo das benfeitorias}

O custo da edificação do estudo de caso foi determinado por meio do método da quantificação do custo, como descrito a seguir.

\subsubsection{Orçamento analítico}

Para realização do orçamento analítico foram levantadas as quantidades dos serviços nos projetos da edificação, elaboradas as composições de custos unitários com base na TCPO 13 (2010) e levantados os preços dos insumos na tabela do SINAPI (2018).

Após a determinação do custo total da obra foi calculado o preço de venda. Para isso foram adicionadas as taxas de BDI (Benefícios e Despesas Indiretas) de acordo com informações fornecidas no Acórdão $\mathrm{n}^{\circ} 2622$ do Tribunal de Contas da União (TCU) (BRASIL, 2013), ou seja, 4\%, 1,23\% e 7,40\% para o lucro, despesas administrativas e financeiras, respectivamente.

\subsubsection{Uso do CUB}

A estimativa de custo por meio do CUB foi realizada em duas etapas:

a) Primeira metodologia do CUB

Foi utilizado o CUB sem desoneração para a região Oeste do Paraná, referente ao mês de maio de 2018, para a tipologia prédio popular de padrão baixo (CUB PP - 4) que se constituiu como a mais compatível em relação a edificação avaliada.

De acordo com Abunahman (1999), essa metodologia visa incluir ao CUB os custos não considerados no mesmo, como elevadores, instalações, custos administrativos, financeiros e lucros. Esses custos adicionais foram obtidos por meio do orçamento detalhado.

Após a determinação do valor do CUB corrigido, na unidade de $\mathrm{R} \$ / \mathrm{m}^{2}$, esse foi multiplicado pela área equivalente do imóvel, obtendo-se assim o custo total da edificação.

b) Segunda metodologia do CUB

Essa metodologia se aplica quando só se possui o valor do CUB para o projeto-padrão R8 - N (residencial de oito pavimentos e padrão normal de acabamento) e a edificação a ser avaliada não possui a mesma configuração.

Segundo Lopes e Alonso (2014), consiste na aplicação de um coeficiente de ajuste ao CUB, em função da tipologia de obra a ser avaliada, a fim de fazer a transição do custo do padrão construtivo $\mathrm{R} 8$ - N para a benfeitoria analisada. 
Sendo assim, o custo da construção foi obtido pela multiplicação do valor do CUB corrigido (com a aplicação do coeficiente) pela área equivalente de construção.

\subsection{Depreciação da construção}

Após a determinação dos custos da edificação pelas três metodologias citadas, foi efetuada a depreciação para obter o valor atual, com base no estado de conversação e idade da mesma. Para isso foi utilizado o método de Ross combinado ao modelo matemático de Heidecke.

Em seguida foi adicionado ao valor da construção, o valor do terreno e efetuada a multiplicação pelo fator de comercialização 0,95 (por se tratar de uma obra de apartamentos residenciais, na qual há procura razoável e boas possibilidades de venda). Em geral, os profissionais da área consideram esse fator entre 0,85 e 1, dependendo das possibilidades de venda.

\section{RESULTADOS E DISCUSSÕES}

Após a determinação do valor do terreno e dos valores da construção depreciados, para cada uma das metodologias analisadas, foi possível aplicar o método evolutivo, sendo obtidos os valores apresentados no Quadro 2.

\section{Quadro 2 - Valor do Imóvel - Método Evolutivo}

\begin{tabular}{|l|c|c|c|}
\hline \multicolumn{1}{|c|}{ Valores } & $\begin{array}{c}\text { Orçamento } \\
\text { analítico }\end{array}$ & $\begin{array}{c}\text { CUB - Primeira } \\
\text { Metodologia }\end{array}$ & $\begin{array}{c}\text { CUB - Segunda } \\
\text { Metodologia }\end{array}$ \\
\hline Valor mínimo do terreno & $\mathrm{R} \$ 496.827,54$ & $\mathrm{R} \$ 496.827,54$ & $\mathrm{R} \$ 496.827,54$ \\
\hline Valor médio do terreno & $\mathrm{R} \$ 522.524,97$ & $\mathrm{R} \$ 522.524,97$ & $\mathrm{R} \$ 522.524,97$ \\
\hline Valor máximo do terreno & $\mathrm{R} \$ 548.222,39$ & $\mathrm{R} \$ 548.222,39$ & $\mathrm{R} \$ 548.222,39$ \\
\hline Valor da construção depreciado & $\mathrm{R} \$ 1.676 .961,97$ & $\mathrm{R} \$ 1.808 .390,83$ & $\mathrm{R} \$ 1.898 .306,21$ \\
\hline Valor total mínimo & $\mathrm{R} \$ 2.065 .100,03$ & $\mathrm{R} \$ 2.189 .957,45$ & $\mathrm{R} \$ 2.275 .377,06$ \\
\hline Valor total médio & $\mathrm{R} \$ 2.089 .512,59$ & $\mathrm{R} \$ 2.214 .370,01$ & $\mathrm{R} \$ 2.299 .789,62$ \\
\hline Valor total máximo & $\mathrm{R} \$ 2.113 .925,14$ & $\mathrm{R} \$ 2.238 .782,56$ & $\mathrm{R} \$ 2.324 .202,17$ \\
\hline
\end{tabular}

Fonte: AUTORES 2019

Por meio da análise dos resultados apresentados no Quadro 2, é possível perceber que para a primeira metodologia do CUB, obteve-se uma diferença que equivale a $6 \%$ de aumento em relação ao orçamento analítico. Já no caso da segunda metodologia, constatou-se um aumento de $10 \%$ no valor.

Em vista disso, pode-se afirmar que o CUB, em suas duas metodologias, superestima o custo em relação ao orçamento analítico.

Na primeira metodologia, por usar um valor de CUB de uma tipologia semelhante a da edificação analisada, sendo acrescentados os itens não considerados pelo mesmo, os resultados foram mais próximos do orçamento detalhado. Mesmo assim, no CUB PP 4, são considerados alguns itens, como guarita e depósito com banheiro, que a obra em estudo não contém.

Já para o padrão R8 - N, há uma série de elementos que a obra não contempla, como elevadores, salão de festas, copa, banheiros e garagem coberta. A configuração dos apartamentos também é diferente, com dois quartos e uma suíte. Isso justifica de certa forma o valor mais alto obtido, associado com o fator de correção genérico, utilizado na 
segunda metodologia de uso do CUB. Isso reforça a ideia de que o CUB apenas deve ser utilizado para estimativas preliminares, e não para definição do custo da edificação e posterior determinação do valor dos imóveis, como demonstrado neste trabalho.

Apesar da segunda metodologia do uso do CUB ser a mais rápida e ser utilizada por profissionais da área de avaliação de imóveis, é a que apresentou a maior variação em relação ao orçamento detalhado. Isso se dá pelo fato de que a correção no valor do CUB $\mathrm{R} 8$ - N é feita apenas pela multiplicação por um coeficiente fixo, em função do padrão construtivo, sem considerar as peculiaridades da obra.

\section{CONSIDERAÇÕES FINAIS}

Embora seja mais preciso, o orçamento analítico nem sempre é usado por todos os profissionais da área de avaliação de imóveis. Em função disso, muitos utilizam a estimativa de custos por meio do CUB. Porém, com a realização deste trabalho foi possível perceber que o valor do imóvel obtido com as duas metodologias de utilização do CUB apresentadas, foi de $6 \%$ e $10 \%$ superior ao valor obtido por meio do orçamento detalhado.

Vale destacar que, embora neste trabalho tenha sido apresentado o uso do CUB para determinação do custo das benfeitorias no método evolutivo, o mesmo deve ser utilizado apenas para estudos preliminares, nas etapas onde os projetos da edificação ainda não foram desenvolvidos, e é necessário efetuar uma estimativa de custo, como para análise de viabilidade.

Porém, como o procedimento é utilizado com frequência para avaliação de imóveis, este trabalho teve como objetivo apresentar as diferenças encontradas, de forma que os profissionais possam se conscientizar de que somente o uso do orçamento detalhado, com base nos projetos e especificações da edificação avaliada, pode conduzir aos valores mais adequados para os imóveis.

\section{REFERÊNCIAS}

ABUNAHMAN, Sérgio Antônio. Curso básico de engenharia legal e de avaliações. São Paulo, SP: Pini, 1999. 301 p.

ARAÚJO, Marcelo Bernardino; REBOLEDO, Alexandre. Análise comparativa de orçamentos de custos: um estudo de caso. Revista Sinergia, São Paulo, v. 19, n. 2, p. 106-114, jul./dez. 2018

ASSOCIAÇÃO BRASILEIRA DE NORMAS TÉCNICAS (ABNT). NBR 12721: avaliação de custos unitários de construção para incorporação imobiliária e outras disposições para condomínios: edifícios - procedimento. São Paulo, 2006.

ASSOCIAÇÃO BRASILEIRA DE NORMAS TÉCNICAS (ABNT). NBR 14653-1: avaliação de bens: parte 1: procedimento gerais. São Paulo, 2001.

ASSOCIAÇÃO BRASILEIRA DE NORMAS TÉCNICAS (ABNT). NBR 14653-2: avaliação de bens: parte 2: imóveis urbanos. São Paulo, 2011.

BRASIL. Tribunal de Contas da União. Acórdão No 2622/2013 - TCU - Plenário. Brasília, 25 set. 2013. Disponível em: <http://licitacoes.ufsc.br/files/2014/10/Ac\%C3\%B3rd\%C3\%A3o2622-2013-BDI.pdf>. Acesso em: 25 abr. 2018. 
SIBRAGEC - ELAGEC 2019 - de 23 a 25 de Outubro - LONDRINA - PR

COELHO, Mauro Celso Vicente. Construção de intervalo de confiança na avaliação pelo método evolutivo com uso de técnica de Monte Carlo. In: XIV COBREAP - CONGRESSO BRASILEIRO DE ENGENHARIA DE AVALIAÇÕES E PERÍCIAS. IBAPE/BA.2007.

FERNANDES, Paula Monteiro de et al. Um estudo comparativo entre o orçamento por composição de custo unitário e o orçamento por estimativa utilizando o índice da Sinduscon CUB/M². Revista Pensar Engenharia, v. 5, n. 2, p. 1-9, jul. 2017. Disponível em: $<$ http://revistapensar.com.br/engenharia/pasta_upload/artigos/a200.pdf $>$. Acesso em 25 mar. 2018.

FLORENCIO, Lutemberg de Araújo. Avaliação de imóveis em massa com base em modelos Gamls. In: XVI COBREAP - CONGRESSO BRASILEIRO DE ENGENHARIA DE AVALIAÇÕES E PERÍCIAS - IBAPE/AM - 2011.

KNOLSEISEN, Patrícia Cecília; LIBRELOTTO, Lisiane Ilha; JUNGLES, Antônio Edésio. Análise comparativa entre orçamento expedito e estimativa de custos através do custo unitário básico: um estudo de caso. In: ENCONTRO NACIONAL DE ENGENHARIA DE PRODUÇÃO, Salvador, 2001. Anais eletrônicos... Salvador, 2001. 8 p. Disponível em: <http://www.abepro.org.br/biblioteca/ENEGEP2001_TR19_0386.pdf>. Acesso em: 25 mar. 2012.

LOPES, José Tarcísio Doubek; ALONSO, Nelson Roberto Pereira. Avaliação de benfeitorias urbanas. In: INSTITUTO BRASILEIRO DE AVALIAÇÕES E PERÍCIAS DE ENGENHARIA DE SÃO PAULO (IBAPE). Engenharia de avaliações. 2. ed. São Paulo: Ed. Universitária de Direito, 2014. v. 1, p. 273-331.

MATTOS, Aldo Dórea. Como preparar orçamentos de obras: dicas para orçamentistas, estudos de caso, exemplos. São Paulo: Pini, 2006.

SISTEMA NACIONAL DE PESQUISA DE CUSTOS E ÍNDICES DA CONSTRUÇÃO CIVIL (SINAPI). O que SINAPI. Disponível em: <http://www.caixa.gov.br/poderpublico/apoio-poder-publico/sinapi/Paginas/default.aspx>. Acesso em: 02 maio 2018.

TCPO 13: tabelas de composições de preços para orçamentos. 14. ed. São Paulo: Pini, 2010. 